\title{
Drug molecules for flexible organic crystals
}

\author{
Meijuan Cao ${ }^{1,2}$, Ketao Chen ${ }^{2}$, Jasjit Kaur ${ }^{2}$ and Hai-Feng Ji ${ }^{2 *}$ \\ ${ }^{1}$ Beijing Engineering Research Center of Printed Electronics, School of Printing and Packaging Engineering, Beijing Institute of Graphic Communication, Beijing, \\ 102600, China \\ ${ }^{2}$ Department of Chemistry, Drexel University, Philadelphia PA 19104, USA
}

\section{Opinion}

Molecular organic crystals are considered to be rigid and fragile. Recently, flexible crystals (also termed plastically bendable crystals) have been reported to possess a good elasticity. As one example, the flexibility of a biocrystal of clofazimine (CFZ) (Figure 1) synthesized in macrophage showed photoelastic effects [1]. CFZ was used as a drug molecule in combination with rifampicin and dapsone to treat leprosy, but later the crystal-like aggregates of CFZ were observed in the intestinal mucosa, liver, spleen, and lymph nodes of patients on CFZ [2]. CFZ may be the first drug molecule that is capable to aggregate to form flexible organic or molecular crystals. While the aggregation will have a negative impact on human health, the flexible crystals have potential applications in many other fields. It is of interest to understand what kind of molecules could form flexible crystals.

A quick survey shows that two major types of molecular organic crystals have been reported; Molecules are held together via aromatic $\pi$ stacking and van de Waals interactions, and via hydrogen-bonded core $\pi$ stacking and van der Waals interactions.

\section{Systems based on aromatic $\pi$ stacking and van de Waals interactions}

Structures of several molecules that form this type of crystals are also shown in Figure 1.

In the crystals made of 4,7-dibromo-2,1-3-benzothiadiazole (DBBT) [3], the crystals showed a slip-stacked $\pi-\pi$ overlap of two DBBT molecules along the wire direction. XRD confirmed that slight sliding of the DBBT molecules occurred, which resulted in the angle and distance changes between molecules in the J-aggregates when bending (Figure 2).

Krishna, et al. [4] reported two crystals made of $\mathrm{N}$-substituted naphthalene diimide (NDIs) or (E)-2-((4-halophenylimino)methyl)6-methoxyphenol. Based on the XRD data and SEM images, they concluded that the flexibility of the crystals originated from smooth sliding of molecular sheets because of the weak interlayer van der Waal forces, such as X...X or R...R interactions. The sliding only resulted in slightly different lattice orientations without fracture of the crystals.

For crystals made of copper(II) acetylacetonate $\left(\mathrm{Cu}(\mathrm{acac})_{2}\right)$ [5], Worthy, et al. showed that the molecules in the crystals can reversibly rotate under mechanical compression, which contribute to the elasticity of the blue crystalline wires.

Organic crystals made of polyhalogenated n-benzylideneanilines, such as 2,3-Dichlorobenzylidine-4-bromoaniline, also show high flexibility. In this molecule, n-benzylideneaniline is the core and halogens provide the structural buffers for the crystals to deform during bending [6].

In summary, in all these flexible molecular crystals, the core moieties of the molecules self-assembled in slip-stacked $\pi$ stacking, typically in the form of J-aggregation. This $\pi$ stacking of the building blocks is the backbone of the one dimensional crystals. The alkyl (spherical but not leaner long chain) or halogen groups on the core moieties contribute to the shear sliding property of the flexible crystals without breaking.

\section{Systems based on $\pi$ stacking of hydrogen-bonded cores and van de Waals interactions}

Besides the aromatic $\pi$ stacking system, Krishna, et al. also reported the design of flexible crystals based on $\pi$ stacking of hydrogen bonded cores and van der Waals interactions [4].

This system is similar to the first, but the cores are built via hydrogel bonding between two molecules (Figure 3). Halogens or spherical alkyl groups form the slipping planes that allow the crystals to bend without fracture. It is noted that extra groups on the core that can form more hydrogen bonds should be avoided in order to facilitate the flexibility of crystals.

Magdalena, et al. reported flexible crystals from a family of haloimidazole derivatives. The elasticity of the crystals was due to the halogenated bonding [7]. The molecules are aligned face to face, i.e. H-aggregation.

The crystals of $\mathrm{N}$-capped diphenylalanine is another example that the alkyl groups provide the weak interactions between the selfassembled rigid layers as studied using Density functional theory calculations with high-resolution scanning electron microscopy [8].

${ }^{\star}$ Correspondence to: Ji HF, Department of Chemistry, Drexel University, Philadelphia PA 19104, USA, Tel: 01-215-895-2562; Fax: 01215-895-1265; Email: hj56@drexel.edu

Special Issue: Nanotechnology: Challenges and Perspectives in Medicine

Dr. Federica Valentini

Department of Sciences and Chemical Technologies

Tor Vergata University

Italy

Maurizio Talamo

Professor

Department of Enterprise Engineering

Italy

Received: October 18, 2018; Accepted: October 29, 2018; Published: October 31,2018 


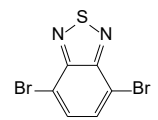

4,7-Dibromo-2,1,3benzothiadiazole $\mathrm{CH}_{3}$ or

$\mathrm{N}$-substituted naphthalene diimides (NDIs)

Figure 1. Structures of several chemicals for the flexible crystals that are made of aromatic $\pi$ stacking cores and van der Waals groups

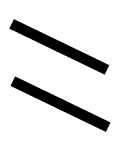

\section{Bending}

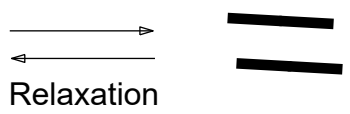

Figure 2. Slight sliding of the DBBT molecules
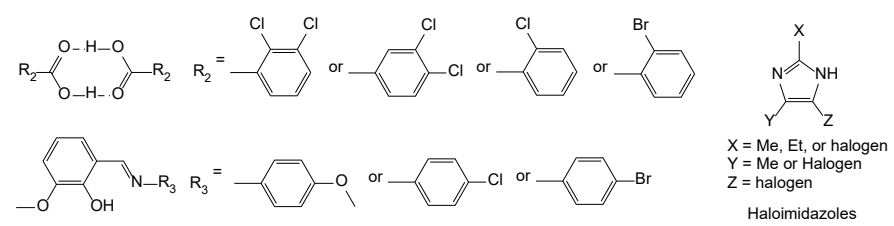

Figure 3. Flexible crystals made of $\pi$ stacking of hydrogen-bonded cores and van der Waals groups

Co-crystals that combine the properties of two molecules in the crystalline wires can also convert brittle crystals to their elastic counterpart as demonstrated by Desiraju, et al. [9].

\section{Potential applications and possible drug molecules for the flexible crystals}

In general, conductive polymers are flexible, but their conductivity is relatively low because of their amorphous character, increasing crystallinity of the polymers can increase the conductivity, but the polymers become more fragile. Thus, the flexible organic crystals have the potential to bridge the gap in order to develop more conductive organic-based flexible electronics and optoelectronics. Examples include flexible ferroelectric crystals,[7] flexible fluorescent emitters or waveguide $[10,11]$, fluorescent elastic organic single crystals, [12,13] crystals for telecommunication [14], sensors [15-17] etc.

Again, CFZ might be the first drug molecules that may form flexible organic crystals. Since many drug molecules have a structure that combines an aromatic core for $\pi$-stacking and $\mathrm{R}$ or halogen groups that allow shear sliding in the case of bending, it is of interest to investigate whether many drug molecules can form organic flexible crystals.

\section{References}

1. Horstman EM (2017) Elasticity in Macrophage-Synthesized Biocrystals. Angew Chem Inter Ed 56: 1815-1819.

2. Baik J, Rosania GR (2011) Molecular Imaging of Intracellular Drug-Membrane Aggregate Formation. Mol Pharm 8: 1742-1749.

3. Hayashi S, Koizumi T, Kamiya N (2017) Elastic Bending Flexibility of a Fluorescent Organic Single Crystal: New Aspects of the Commonly Used Building Block 4,7-Dibromo-2,1,3-benzothiadiazole. Cryst Growth Des 17: 6158-6162.

4. Krishna GR (2016) Mechanically Flexible Organic Crystals Achieved by Introducing Weak Interactions in Structure: Supramolecular Shape Synthons. J Amer Chem Soc 138: 13561-13567.

5. Worthy A (2017) Atomic resolution of structural changes in elastic crystals of copper(II) acetylacetonate. Nature Chem 10: 65 .

6. Ghosh S, et al. (2015) Designing Elastic Organic Crystals: Highly Flexible Polyhalogenated N-Benzylideneanilines. Angew Chem Inter Ed 54: 2674-2678.

7. Owczarek M, et al. (2016) Flexible ferroelectric organic crystals. Nature Comm 7: 13108.

8. Adler-Abramovich L, et al. (2018) Bioinspired Flexible and Tough Layered Peptide Crystals. Adv Mater 30: 1704551.

9. Subhankar Sahaa GRD (2016) Using structural modularity in cocrystals to engineer properties: elasticity. Chem Comm 56: 7676

10. Hayashi S, Yamamoto SY, Takeuchi D, Ie Y, Takagi K (2016) Creating Elastic Organic Crystals of $\pi$-Conjugated Molecules with Bending Mechanofluorochromism and Flexible Optical Waveguide. Angew Chem Inter Ed in press.

11. Liu H (2018) Highly Elastic Organic Crystals for Flexible Optical Waveguides. Angew Chem Inter Ed 57: 8448-8452.

12. Hayashi S (2017) Fluorescent organic single crystals with elastic bending flexibility 1,4-bis(thien-2-yl)-2,3,5,6-tetrafluorobenzene derivatives. Sci Reports 7: 9453.

13. Hayashi S, Koizumi T, (2016) Elastic Organic Crystals of a Fluorescent $\pi$-Conjugated Molecule. Angew Chem Inter Ed 55: 2701-2704.

14. Bisri SZ (2012) Organic Single-Crystal Light-Emitting Transistor Coupling with Optical Feedback Resonators. Sci Reports 2: 985.

15. Wang X, Liu Z, Zhang T (2017) Flexible Sensing Electronics for Wearable/Attachable Health Monitoring. Small 13: 1602790.

16. Bansal AK, et al. (2015) Wearable Organic Optoelectronic Sensors for Medicine. Adv Mater 27: 7638-7644.

17. Xu N, Powell DR, Richter-Addo GB (2011) Nitrosylation in a Crystal: Remarkable Movements of Iron Porphyrins Upon Binding of Nitric Oxide. Angew Chem Inter Ed 50: 9694-9696.

Copyright: (C2018 Cao M. This is an open-access article distributed under the terms of the Creative Commons Attribution License, which permits unrestricted use, distribution, and reproduction in any medium, provided the original author and source are credited. 